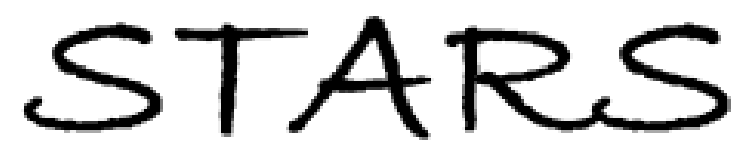

University of Central Florida

STARS

$1-1-1999$

\title{
Cryogenic stabilization of high vapor pressure samples for surface analysis under ultrahigh vacuum conditions
}

\author{
J. J. Bruckner \\ K. Wozniak \\ S. Hardcastle \\ A. Sklyarov \\ S. Seal \\ University of Central Florida
}

See next page for additional authors

Find similar works at: https://stars.library.ucf.edu/facultybib1990

University of Central Florida Libraries http://library.ucf.edu

This Article is brought to you for free and open access by the Faculty Bibliography at STARS. It has been accepted for inclusion in Faculty Bibliography 1990s by an authorized administrator of STARS. For more information, please contactSTARS@ucf.edu.

\section{Recommended Citation}

Bruckner, J. J.; Wozniak, K.; Hardcastle, S.; Sklyarov, A.; Seal, S.; and Barr, T. L., "Cryogenic stabilization of high vapor pressure samples for surface analysis under ultrahigh vacuum conditions" (1999). Faculty Bibliography 1990s. 2567.

https://stars.library.ucf.edu/facultybib1990/2567

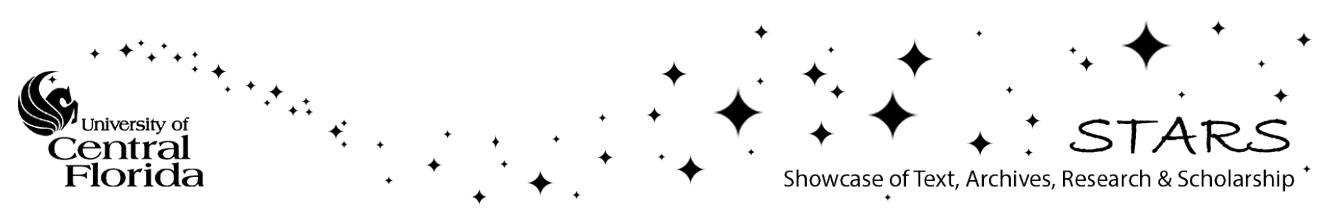




\section{Authors}

J. J. Bruckner, K. Wozniak, S. Hardcastle, A. Sklyarov, S. Seal, and T. L. Barr 
Cryogenic stabilization of high vapor pressure samples for surface analysis under ultrahigh vacuum conditions

\author{
J. J. Bruckner, K. Wozniak, S. Hardcastle, A. Sklyarov, S. Seal, and T. L. Barr
}

Citation: Journal of Vacuum Science \& Technology A 17, 2668 (1999); doi: 10.1116/1.581928

View online: https://doi.org/10.1116/1.581928

View Table of Contents: https://avs.scitation.org/toc/jva/17/5

Published by the American Vacuum Society

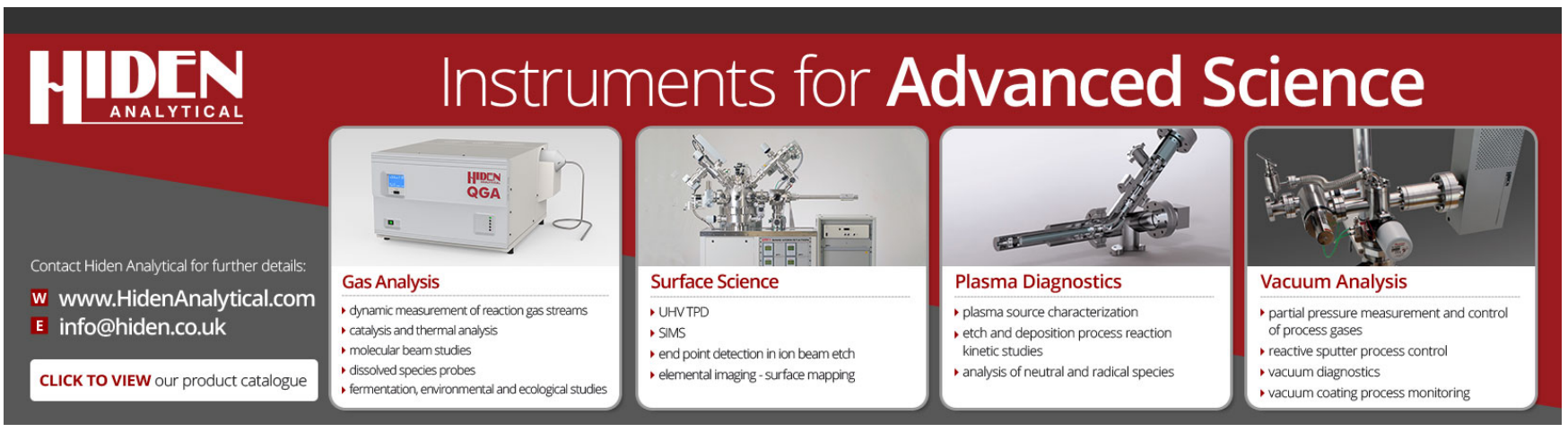




\title{
Cryogenic stabilization of high vapor pressure samples for surface analysis under ultrahigh vacuum conditions
}

\author{
J. J. Bruckner ${ }^{\mathrm{a})}$ \\ Wilson, Sonsini, Goodrich, and Rosati, Professional Corporation, Palo Alto, California 94304 \\ K. Wozniak \\ Chemistry Department, Warsaw University, ul. Pasteura 102093 Warsaw, Poland \\ S. Hardcastle and A. Sklyarov \\ Advanced Analytical Facility, University of Wisconsin-Milwaukee, Milwaukee, Wisconsin 53201 \\ S. Seal \\ Advanced Materials Processing and Analysis Center, Department of Mechanical, Materials and Aerospace \\ Engineering, University of Central Florida, Orlando, Florida 32816 \\ T. L. Barr \\ Department of Materials and Laboratory for Surface Studies, University of Wisconsin-Milwaukee, \\ Milwaukee, Wisconsin 53201
}

(Received 12 March 1998; accepted 11 June 1999)

\begin{abstract}
A novel form of ultrahigh vacuum (UHV) cryogenic stabilization has been used to obtain high-resolution x-ray photoelectron spectroscopy (XPS) data from a complex amine, 1,8-bis(dimethylamino)naphthalene, whose solid phase exhibited at room temperature an unacceptably high rate of sublimation. Protonated versions of the amine exhibit hydrogen bonding. Electron spectroscopy for chemical analysis chemical shifts can be used to describe the strength and asymmetry of hydrogen bonding formed in proton sponge complexes. Analyzing the binding energy shifts of $\mathrm{N}(1 s)$ induced by the presence of this hydrogen bonding required obtaining corresponding XPS spectra from the nonprotonated (reference) sample, but the reference sample sublimes under even moderate vacuum conditions. The combined results suggest that other high vapor pressure materials, particularly those that were previously considered to be too corrosive for routine analysis, can be cryogenically stabilized for surface analysis under similar UHV conditions. (C) 1999 American Vacuum Society. [S0734-2101(99)09605-0]
\end{abstract}

\section{INTRODUCTION}

X-ray photoelectron spectroscopy (XPS), also known as electron spectroscopy for chemical analysis (ESCA), provides useful electron density data that are indicative of the chemical state of the atomic elements in the surface region of a solid sample. XPS is therefore a surface analysis technique that analyzes photoelectrons emitted from the outer 10-80 of the surface of a sample. ${ }^{1}$ Generally in this region, there will be some adsorption of, and chemical involvement with, air induced species including adsorbed carbonaceous species (particularly hydrocarbons), which can be designated as adventitious carbon. The latter are always found on air exposed surfaces. The resulting adsorbed oxides and carbonaceous species are relatively inert to more deleterious environmental effects, thus providing a modest form of "natural" passivation that can be useful to protect some samples. ${ }^{2}$

The nature of these environmental effects may usually be recognized and separated from the detected properties of the experimental surface of interest. Moreover, the persistent adventitious hydrocarbons often provide a basis for establishing the photoelectron binding energy scale needed for XPS chemical analysis, especially when the sample of material of interest exhibits charging (i.e., a lack of coupling to the

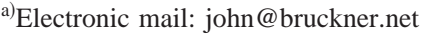

Fermi edge of the instrument) due to low electronic conductivity. ${ }^{3,4}$

Many spectroscopies cannot directly detect the lowest atomic number elements. One of the most often employed negative statements concerning electron spectroscopies, [i.e., both XPS and Auger electron spectroscopy (AES)] is that they cannot be employed to detect hydrogen. In the minds of many this contention has often been overextended to the statement that XPS is blind to the effects of hydrogen. This is incorrect. Such overgeneralization ignores the well known fact that many of the chemical determinations in XPS are based on the binding energy shifts induced into the spectra of an atom $\mathrm{A}$, for example, carbon, by another atom $\mathrm{B}$, for example, hydrogen, in an $\mathrm{A}_{x} \mathrm{~B}_{y}$ system. Thus, while it is true that hydrogen cannot be directly detected by XPS, most surface scientists are aware that the effects of hydrogen can be detected by XPS by observing the effects of hydrogen in compounds on its adjacent atoms (e.g., on the carbon spectra). ${ }^{5-8}$ In the present case, the effect of hydrogen bonding was detected by XPS by observing the progressive effects of that bonding on the nitrogen $(1 s)$ spectra of the various complex amines involved.

All commercial XPS systems require that the sample be held in ultrahigh vacuum (UHV) of approximately $1(10)^{-8}$ Torr or better. However, many materials that are solid at 
standard temperature and pressure (STP) vaporize by sublimation at pressures well above $1(10)^{-8}$ Torr, thereby prohibiting the use of conventional, room temperature XPS techniques for chemical state analysis of such volatile materials.

It has been demonstrated that with the use of differential pumping XPS analysis can be achieved at pressures as high as $1(10)^{-3}$ Torr. However, most XPS systems are not equipped with this intricate and often costly capability. Further, at the typical XPS operating temperature of approximately $300 \mathrm{~K}$, many high vapor pressure materials of interest may vaporize too much at pressures above $1(10)^{-3}$ Torr in order to successfully employ conventional pumping to maintain these pressures.

A novel route around this dilemma is to cool such volatile sample materials to a sufficiently low temperature (e.g., liquid nitrogen temperatures) before vacuum conditions are imposed. Cooling the sample sufficiently allows the investigator to take advantage of the resulting lower free energy often reducing the vaporization rate below a level compatible with the pumping system, thus stabilizing an otherwise volatile material as a solid, despite high vacuum and, in many cases, even UHV conditions.

However, in most XPS systems, the cooling subsystem is designed to cool a sample only after it has already been situated in a preparation and/or analysis chamber under UHV conditions. If, due to the requirement for stabilization, the sample must be frozen before entry into the UHV analysis chamber, the low temperatures of a liquid nitrogen regime can wreak havoc with certain aspects of the sample handling and transfer components (e.g., entry seals) due to dissimilarities in thermal expansion coefficients. More particularly, if the sample must be cooled in an introduction chamber to achieve stabilization prior to being moved into an UHV preparation chamber, and then moved again into the UHV analysis chamber, the process of transferring the frozen sample can be problematic. Specifically, the UHV seals can be adversely affected by the low temperature regime (e.g., VG O rings). An example of such an adverse effect includes differential shrinkage of gasket materials. Further, in the case of polymeric gaskets, their substantially increased viscosity is also an adverse effect. The latter effect can be particularly onerous where the polymeric gaskets themselves are moving parts during the sample handling dynamics (e.g., HP gaskets).

In the present case, these problems are addressed by modifying the usual pumping protocol to one in which the preparation chamber is first backfilled to atmospheric pressure with argon and the sample is then moved from the introduction chamber to the preparation chamber prior to imposing UHV conditions.

\section{EXPERIMENT}

The high vapor pressure sample that was stabilized with the extended pumping protocol was nonprotonated 1,8bis(dimethylamino)naphthalene (DMAN). Also known as $N, N, N, N^{\prime}$ tetramethyl-1,8-naphthalenediamine, this material

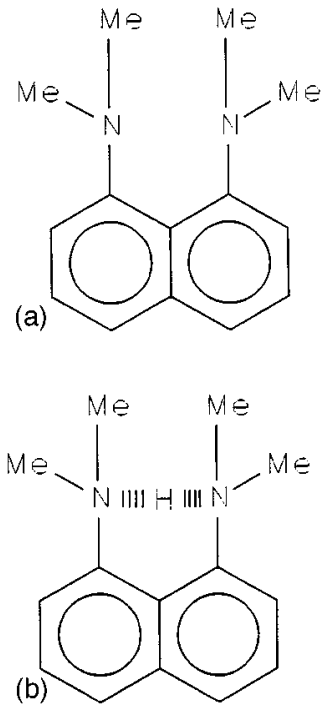

FIG. 1. (a) Nonprotonated DMAN. (b) Protonated DMAN.

has been assigned Chemical Abstracts Service No. 20734$58-1$. DMAN has a melting point from 49 to $51^{\circ} \mathrm{C}$ at a pressure of 760 Torr. DMAN is readily available commercially from Lancaster Synthesis Ltd., Windham, NH. In Fig. 1(a), the nonprotonated species of DMAN is illustrated. DMAN is a strong base that due to its propensity to incorporate $\mathrm{H}^{+}$units is often labeled as a proton sponge. ${ }^{9,10}$

The protonated species of DMAN include the shared bonding of one hydrogen atom. ${ }^{11}$ In Fig. 1(b), the hydrogen of interest is depicted between the two aniline/amino type nitrogen atoms. This hydrogen is known to bind between the two aniline/amino type nitrogen atoms into structures that have been confirmed by nuclear magnetic resonance (NMR) and $\mathrm{x}$-ray diffraction (XRD) studies. ${ }^{6,8,10,12-16}$

For those involved in studies of proton sponges it is well known (both from theoretical and experimental studies) that there is only one hydrogen in the $[\mathrm{N}-\mathrm{H} \ldots \mathrm{N}]^{+}$hydrogen bridge. ${ }^{6-8,10,12,14}$ This has been confirmed by ab initio calculation as well as by numerous solution and solid state NMR results and $\mathrm{x}$-ray and neutron diffraction studies. There is not enough space for two hydrogen atoms in the $[\mathrm{N}-\mathrm{H} \ldots \mathrm{N}]^{+}$ hydrogen bridge although some proton sponge complexes can have disordered $\mathrm{H}$ bonding with the proton jumping over the two sites close to the nitrogen atoms. This is a dynamic situation with the proton tunneling between the two allowed positions. On average this would give two hydrogens/protons with the occupancy factors equal to $50 \%$ located in such a $\mathrm{H}$ bridge but it does not mean that these two hydrogen positions are occupied at the same time. On the contrary, only one of them is occupied. They both are occupied in a statistical manner with equal probability (50\%). However such a disordered $\mathrm{H}$ bond is somewhat of an exception and in general far more often the ordered asymmetric $[\mathrm{N}-\mathrm{H} . . \mathrm{N}]^{+}$hydrogen bridge is found in proton sponge complexes.

Direct proof of the presence of $\mathrm{H}$ in the hydrogen bond comes from solid state NMR which clearly shows the pres- 
ence of only one $\mathrm{H}$ atom with extremely large chemical shifts $(\sim 18 \mathrm{ppm}$ compared with typical values for hydrogen atoms in the range from $\sim 1 \mathrm{ppm}$ for the aliphatic $\mathrm{H}$ attached to carbons, up to 10-12 ppm for protons involved in a typical hydrogen bonding). This extra deshielding of the proton in the proton sponge complexes comes from cooperative interactions and close proximity of the lone electron pairs of the nitrogens atoms.

As we will amplify below, different acids generally exhibit different degrees and positions of the resulting $\mathrm{H}^{+}$incorporation. Variations in the linearity and centricity of the resulting $\mathrm{N}-\mathrm{H}$ bonds are crucial. They depend on the strength of interactions with the closest counterion present in the crystal lattices of the ionic complexes of DMAN. Usually, the closest counterion to the $\mathrm{DMANH}^{+}$cation is located in front of the bulky methyl groups and it has a strong influence on the proton involved in the $[\mathrm{N}-\mathrm{H} \ldots \mathrm{N}]^{+}$hydrogen bridge. A nice manifestation of such secondary interactions are the values of the NHN hydrogen bond angle which is smaller by $\sim 5^{\circ}$ in the crystal complexes than the value obtained from $a b$ initio calculations for the isolated DMANH $^{+}$cation not interacting with any anion. ${ }^{9}$

The present $\mathrm{X}$-ray photoelectron spectroscopy analysis was achieved using a modified Hewlett Packard ESCA spectrometer (HP 5950A). The base pressure of this XPS system is generally on the order of $1(10)^{-9}$ Torr.

This XPS system employs a three Si crystal x-ray monochromator to improve energy resolution and sensitivity. Any sample charging was removed with a specially designed low energy electron flood gun. A nonmonochromatic system would have a more difficult time with such sensitive samples.

This XPS system uses a long cylindrical sample probe to transfer a sample located near the distal tip of the sample probe by sliding the sample probe in toward the center analysis chamber. The proximal end of the sample probe is always exposed to ambient. There is a first vacuum seal between a first pair of Teflon ${ }^{\mathrm{TM}}$ compression rings that are part of the preparation chamber and the outer surface of the sample probe. In addition, there is an UHV seal between a second and third pair of Teflon ${ }^{\mathrm{TM}}$ compression rings that are part of the preparation chamber and the outer surface of the sample probe. The second and third pair of rings are situated between the analysis chamber flange and the first compression ring. In addition there is an UHV isolation valve between the preparation and analysis chambers. This permits the pressure in the preparation chamber to be as high as ambient for sample loading while the analysis chamber remains at UHV.

One form of sample probes available is equipped with a liquid nitrogen cooling system. The liquid nitrogen cooling system controller can maintain a stable temperature as low as $120 \mathrm{~K}$. Liquid nitrogen supplied to an inlet at the proximal end of this probe is routed down its length through a conduit to a region adjacent to the sample holding slots, which are located near the distal tip of the probe. This $\mathrm{N}_{2}$ (liquid) is then routed back up the length of the sample probe through another conduit to an outlet at the proximal end. A solenoid valve controlled by a predetermined setpoint acts to control the flow of liquid nitrogen through the probe. While the compression rings of the UHV seals were designed to maintain UHV when a sample probe is being repositioned for sample loading, they were not designed to maintain UHV during insertion when the probe is at cryogenic temperatures.

Thus, extreme cooling of the sample causes both the sample probe and (if subjected to this cooling) the compression rings to undergo differential shrinkage due to dissimilar thermal coefficients. Meanwhile, the viscosity of the Teflon $^{\mathrm{TM}}$ rings increases substantially due to the lowered temperature. In addition, frost that condenses on the outer surface of the sample probe, which is exposed to ambient moisture, will contribute substantially to difficulties in maintaining UHV as the sample probe is inserted into the analysis chamber. Thus, some other means of sample insertion was necessary.

From previous experience, it was determined that nonprotonated DMAN sublimes under UHV conditions at room temperature. Unfortunately, there is no published phase data for nonprotonated DMAN. In an effort to estimate the ambient temperature sublimation point for the nonprotonated DMAN, a simple bell jar was employed together with a low vacuum mechanical pump. Complete sublimation was found to occur at $2(10)^{-3}$ Torr and approximately $295 \mathrm{~K}$.

A nonprotonated DMAN sample designed for cryogenic stabilization was prepared by grinding the material with a mortar and pestle. The powder was packed into a sample holder that was then inserted into the sample probe near the distal tip of the sample probe in a manner designed to present a smooth sample surface on the Rowland circle provided by the analysis chamber under optimum conditions. ${ }^{2}$

In order to begin the present experiments, the loaded sample probe was inserted into the preparation chamber at ambient pressure and the cooling system was turned on. In view of the fact that no phase information was available for DMAN, it was not known to what temperature the sample would have to be cooled in order to retain the solid state status of the DMAN. The sample was initially cooled to a temperature of $150 \mathrm{~K}$ since this was hoped to be adequate, given the $120 \mathrm{~K}$ minimum for this system under UHV. When the monitored temperature of the sample probe at ambient became stable at $150 \mathrm{~K}$, the mechanical roughing pump was turned on. As the roughing process proceeded, a modest presence of visible frost began to build up on the external surfaces of the sample probe exposed to ambient atmosphere. After approximately $1 \mathrm{~h}$, the pressure in the preparation chamber became low enough so that the diffusion pump was turned on. Based on the observed vacuum level, the sample proceeded to outgas for an extended period of time as the condensation of primarily $\mathrm{H}_{2} \mathrm{O}$ had to be evacuated as well as the $\mathrm{N}_{2}, \mathrm{O}_{2}$, etc.

Meanwhile, liquid nitrogen was fed into the sample probe on an intermittent basis to maintain the low temperature since heat was continuously being transferred from the ambient surroundings to the sample probe. Further, the amount of apparent frost on the sample probe continued to increase.

The temperature setpoint on the temperature controller 


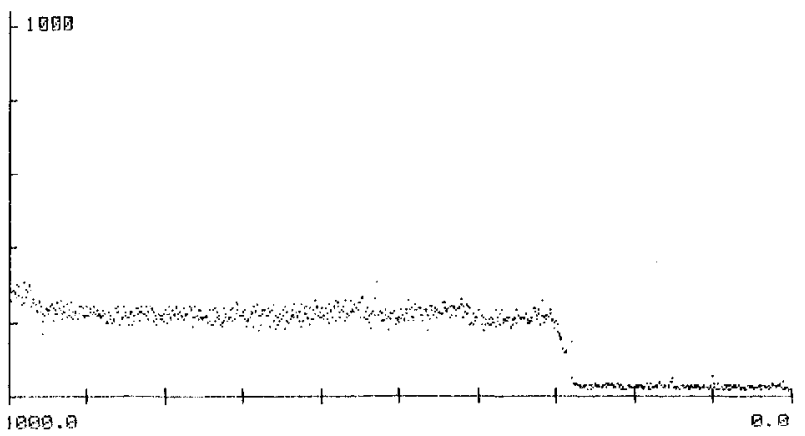

FIG. 2. Survey spectra for nonprotonated DMAN.

was slowly increased to determine the temperature sensitivity of the high vapor pressure sample. At $180 \mathrm{~K}$, an unknown condensed phase formed on the sample probe. At $210 \mathrm{~K}$, the condensed phase appeared to evaporate. The temperature setpoint was raised in $10^{\circ}$ increments to $250 \mathrm{~K}$ and no discernable pressure increase was observed. Only at $270 \mathrm{~K}$ was some pressure increase perceived.

As the preparation chamber was pumped down to UHV, a heat gun was used to melt the frost that continued to accumulate on the sample probe. After it appeared that a suitable and stable UHV pressure had been achieved, the sample was very slowly moved into the analysis chamber. While moving the sample, the pressure in the analysis chamber began to rise. This is believed to have been due to imperfect sealing between the Teflon ${ }^{\mathrm{TM}}$ gasket seals and the sample probe. Therefore, the sample was moved in modest increments of approximately $1 \mathrm{~mm}$ at a time. With each pressure rise, the pressure in the analysis chamber was allowed to stabilize and then the slow incremental advance was repeated.

During data collection, the temperature of the sample was held at approximately $200 \mathrm{~K}$. The pressure in the analysis chamber was approximately $1(10)^{-8}$ Torr during data collection.

After data collection was complete, the sample was taken from the analysis chamber back to the preparation chamber. While moving the sample, the pressure in the analysis chamber began to rise once again. Therefore, the sample was again moved in modest increments of approximately $2 \mathrm{~mm}$ at a time. After each incremental movement, the pressure in the analysis chamber was allowed to stabilize and then the slow incremental retraction was repeated. Finally, the gate valve was closed and the preparation chamber ion pump was turned off.

The preparation chamber was then isolated and backfilled to ambient pressure with argon. The liquid nitrogen supply to the sample probe was then shut off and the sample probe slowly withdrawn from the preparation chamber. By allowing the sample probe to rise to ambient temperature, no undue stress was placed on the ring seals.

The nonprotonated DMAN sample was then removed from the sample probe. Despite all the elaborate pumping and sample movement precautions, it was apparent based on the diminished thickness of the removed sample that a substantial part of the sample material had sublimed.

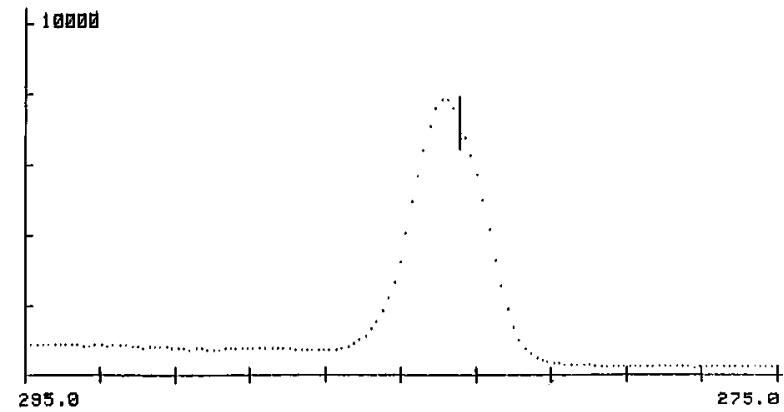

FIG. 3. Carbon $1 s$ spectra for nonprotonated DMAN.

\section{XPS RESULTS AND DISCUSSION}

The XPS results for DMAN reveal a singular binding energy study for nonprotonated DMAN that suggests a reasonably pure version of that material. This has permitted a detailed study of proton induced hydrogen bonding through comparison of the latter with the measured binding energies [particularly those of the $\mathrm{N} 1(s)$ of protonated species of DMAN].

Further, in related fashion we have initiated a detailed study of the influence of hydrogen on the XPS results of select oxide hydroxide, hydrocarbon and hydrogen bonding cases. $^{5}$

Figure 2 illustrates a survey scan of the cryogenically stabilized nonprotonated DMAN sample. Carbon, nitrogen and oxygen peaks are evident. There is no apparent involvement of species such as $\mathrm{F}, \mathrm{Cl}$ and $\mathrm{Br}$.

Figure 3 illustrates the carbon $1 s$ region for the cryogenically stabilized nonprotonated DMAN sample. The shoulder peak on the right in Fig. 3 is assigned to adventitious carbon and/or the hydrocarbon part of the DMAN systems. As a result, all binding energy values were adjusted approximately $1.2 \mathrm{eV}$ up field to be in accord with the standard value of $284.6 \mathrm{eV}$ for these hydrocarbon species. ${ }^{3}$

In Fig. 4 the nitrogen $1 s$ region for the cryogenically stabilized nonprotonated DMAN shows a single, relatively narrow peak, suggesting a singular species with one type of nitrogen. Our best estimate of the $\mathrm{N}(1 s)$ binding energy for nonprotonated DMAN is $399.5 \mathrm{eV}$ based on adventitious

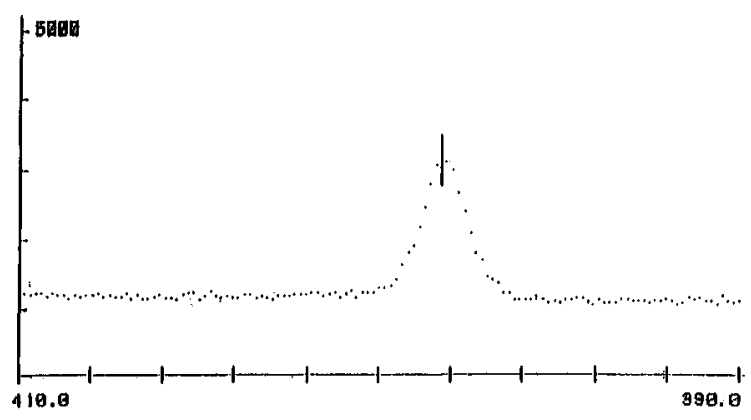

FIG. 4. Nitrogen $1 s$ spectra for nonprotonated DMAN. 


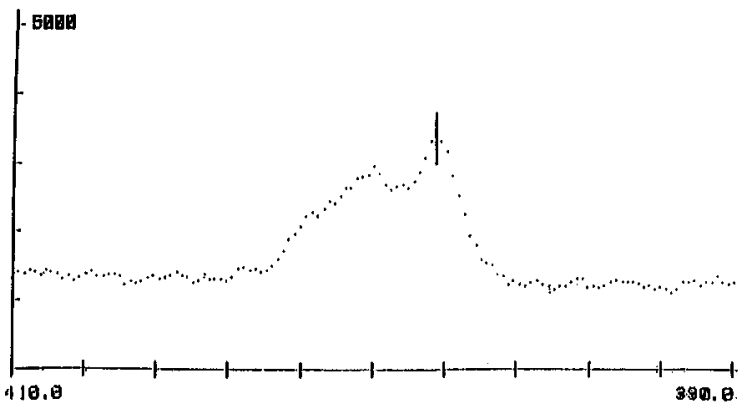

FIG. 5. Nitrogen $1 s$ spectra for $\mathrm{HBr}$ protonated DMAN.

carbon being assigned a binding energy value of $284.6 \mathrm{eV}$. The absence of any significant up-field peaks (i.e., oxidized $\mathrm{H}$ or the hydrogen bonding effect) is evidence of the nonprotonated state of this cryogenically stabilized (reference) sample. The N (1s) binding energy value of $399.5 \mathrm{eV}$ is consistent with the anticipated peak position for the nitrogen in DMAN. ${ }^{7,8}$

In fact, because the DMAN molecule crystallizes in a general position of the orthorhombic $\mathrm{P} 2{ }_{1} 2_{1} 2_{1}$ space group, there are two independent nitrogen atoms present in this structure. Although the isolated molecule of DMAN is symmetric, the DMAN molecule in the crystal lattice seems to be slightly asymmetric due to some asymmetric interactions with the closest crystal environment (the crystal environment of the left half of the DMAN molecule is not the same as the environment of the right half of the moiety). The N (1s) ESCA spectrum of DMAN demonstrates then that such tiny asymmetric interactions are difficult to detect with XPS. Of course, protonation is a far stronger perturbation than the above interactions and this is why its consequences can be more readily detected with ESCA.

In Fig. 5, for comparison, the corresponding nitrogen $1 s$ region for the DMAN species associated with hydrogen bromide is shown. A plurality of up-field peak(s) is clearly evident and this has been shown to be proof of the creation of the protonated state in DMAN. The same signal amplitude and photoelectron binding energy scales as in Fig. 4 are used in Fig. 5, without any relative charge shifting or relative amplitude modifications.

Correspondingly, in Fig. 6, the nitrogen $1 s$ regions from both Figs. 4 and 5 are shown superimposed. It can be seen

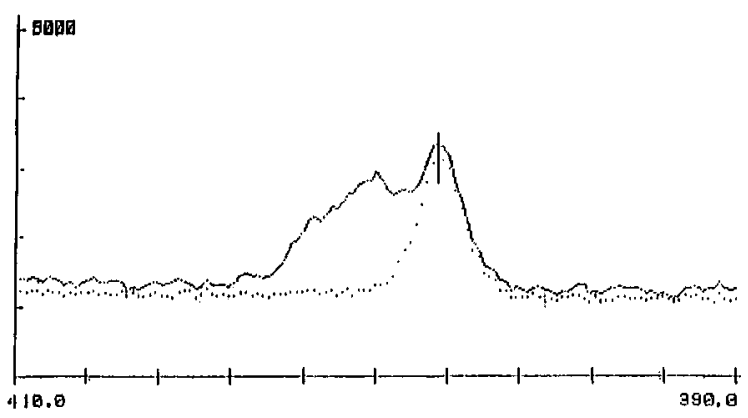

FIG. 6. Overlay of Figs. 4 and 5. that the up-field signals from the protonated species are clearly absent in the reference spectra. The ESCA N (1s) signal coming from the nitrogen atoms involved in the $[\mathrm{N}-\mathrm{H} \ldots \mathrm{N}]^{+}$hydrogen bonding is perturbed by weak interactions with a counterion present in such structures. The counterion form, together with the DMANH ${ }^{+}$cation, forms the crystal structures of the DMAN salts. Such counterions are usually located (determined from x-ray and neutron studies) close to the DMANH ${ }^{+}$cation (in front of the methyl groups). In fact, the $[\mathrm{N}-\mathrm{H} \ldots \mathrm{N}]^{+}$hydrogen bonding also has a minor component, which comes from interactions with this closest counterion, and it might be better to use $[\mathrm{NH} \ldots \mathrm{N}]^{+}$ $\ldots \mathrm{X}^{-}$nomenclature to stress the role of the counterion denoted as $\mathrm{X}^{-}$. In summary, the stronger the acid used to produce the complex, the more electronegative the $\mathrm{X}^{-}$anion, the stronger the interactions of the major component of hydrogen bonding $[\mathrm{NH} \ldots \mathrm{N}]^{+}$with the $\mathrm{X}^{-}$counterion, the larger positive charge induced at the hydrogen involved in the hydrogen bonding and the more electronegative the $\mathrm{N}$ atoms.

There are a number of well established three dimensional structures of DMAN and its complexes. They may be found in the Cambridge Structural Database containing structural data for organic crystals. This Database as well as original papers were used to visualize and examine the structures. According to numerous structures of DMAN complexes, the DMANH + cation is located in such a manner in the crystal lattice that it is accompanied by a counterion that is usually placed in front of the four methyl groups. The anion is located in an asymmetric way-closer to one of the dimethylamino groups. The location of the counterion is due to weak interactions that constitute a minor component of the multicenter $[\mathrm{N}-\mathrm{H} \ldots \mathrm{N}]+\mathrm{H}$ bond.

For example, in a complex of 1,8bis(dimethylamino)naphthalene (DMAN) with 1,2dichlormaliec acid, due to strong electrostatic forces substantially every DMANH+ cation is surrounded by six negatively charged ClMH- moieties and vice versa (see Ref. 6). Any sites in such complexes without adjacent counterions are statistically insignificant.

All these facts are well known. As another example, see Ref. 16.

In a related manner, a series of samples of protonated DMAN were achieved by reaction with a variety of acids. These were analyzed by XPS, as well as by NMR/XRD. ${ }^{6,8,10,12,14}$ The spectroscopic and diffractional data clearly confirm the presence of only one proton in the $[\mathrm{NH} \ldots \mathrm{N}]^{+}$hydrogen bonding that is exhibited by proton sponges. Cryogenic stabilization was not required for these protonated species.

In Fig. 7, the nitrogen $1 s$ XPS spectra for this variety of protonated species of DMAN is shown. The variable nature of the resulting hydrogen bonding is evidenced by the diversity of the up-field range of the peaks (labeled I) at the left of each spectrum. The peak on the right of each these spectra (labeled II) lines up with, and is attributed to, the parent DMAN. ${ }^{7,8}$ Spectral deconvolution in Fig. 7 was accom- 


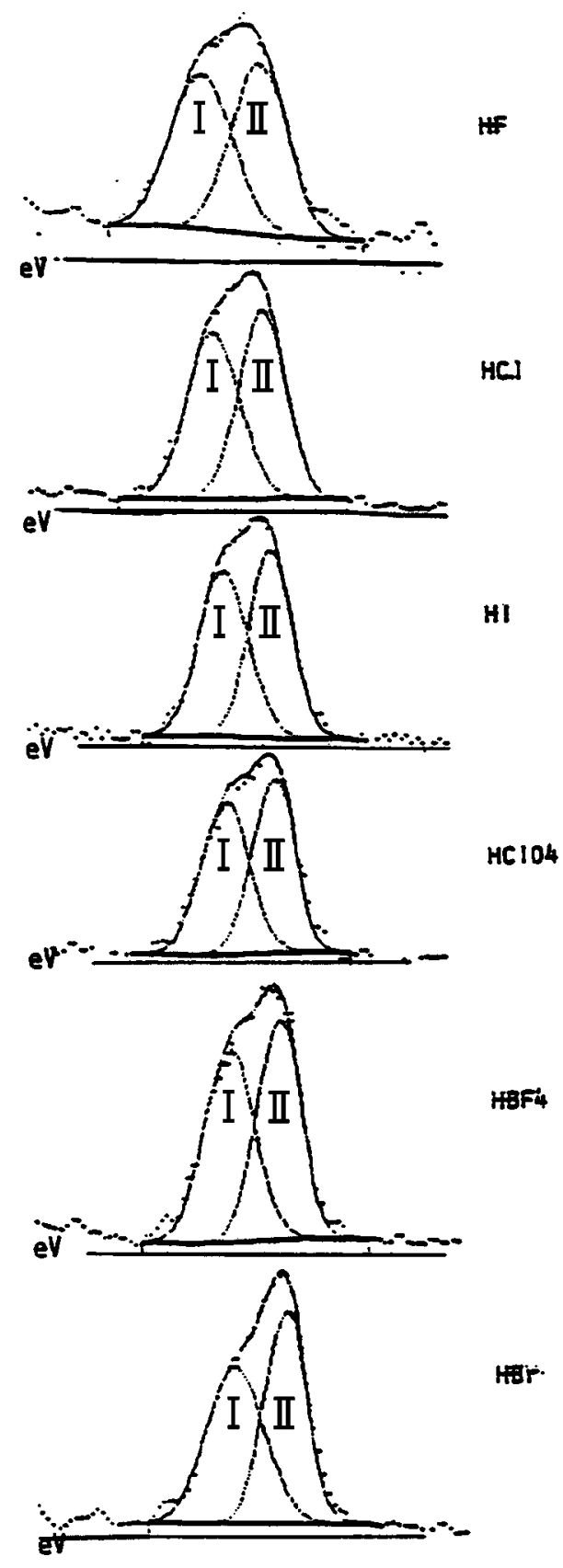

FIG. 7. Nitrogen $1 s$ spectra for six protonated DMAN species.

plished after removal of the Shirley background by minimizing chi squared using a standard peak-fitting software package. All of the core level photo emission peaks were successfully fitted with a Gaussian-Lorentzian distribution. As noted above, the binding energy reference has been made with respect to the carbon $(1 s)$ peak at $284.6 \mathrm{eV}$. Reasons for different shifts depending on $\mathrm{pH}$ are discussed below.

It can be appreciated that the magnitude (relative height) of the hydrogen bonding peak is a recognized function of the relative amount of $\mathrm{H}^{+}$accumulation and, therefore, may be a function of the acidity of the proton donating reactant. In this regard, the height of the hydrogen bonding peak (I) is much greater in the case of highly acidic hydrogen fluoride than in the case of mildly acidic hydrogen bromide. This is not sur-
TABLE I. Nitrogen $1 s$ binding energy (eV) for seven DMAN species. (NA $=$ not available.)

\begin{tabular}{lcc}
\hline \hline \multicolumn{1}{c}{ Material } & Peak I & Peak $\mathrm{II}^{\mathrm{a}}$ \\
\hline DMAN-HF & 403.0 & 399.5 \\
DMAN-HCL & 402.5 & 399.5 \\
DMAN-HI & 402.0 & 399.5 \\
DMAN-HCIO $_{4}$ & 402.0 & 399.5 \\
DMAN-HBF $_{4}$ & 402.0 & 399.5 \\
DMAN-HBr & 402.0 & 399.5 \\
DMAN nonprotonated & NA & 399.5 \\
\hline \hline
\end{tabular}

${ }^{a}$ Based on the adventitious carbon being assigned a binding energy value of $284.6 \mathrm{eV}$.

prising because more acidic donating reactants are more likely to contribute protons to the DMAN matrix. Thus, the higher acidity of the stronger proton donating reactants is evidenced by hydrogen bonding peaks of greater magnitude.

In these cases, hydrogen bonding results in the insertion of $\mathrm{H}^{+}$units into the diamine. The more effective this insertion, the more the system replicates the $3.0+\mathrm{eV}$ shift experienced by $\mathrm{NH}_{3}$ in going to $\mathrm{NH}_{4}^{+}$. It is anticipated that stronger acids are better at realizing this than weaker acids. Stronger acids tend to incorporate the $\mathrm{H}^{+}$more effectively.

Moreover, it can be appreciated that the amplitude (lateral displacement) of the hydrogen bonding shift also appears to be a function of the acidity of the proton donating reactant. In more detail, the binding energy shift for the (I) peak of the highly acidic HF species appears to be approximately $3.5 \mathrm{eV}$. In contrast, the binding energy shift for the (I) peak of the slightly less acidic $\mathrm{HC} 1$ species appears to be approximately $3.0 \mathrm{eV}$. Further, the binding energy shifts for the (I) peaks of the remaining, less acidic species appear to be from approximately 2.5 to approximately $3.0 \mathrm{eV}$. Thus, the higher acidity of the stronger proton donating reactants apparently directly influences the size of the binding energy shift.

Our best estimate for the effect of hydrogen bonding on nitrogen for these proton sponges is the inducement of a binding energy shift from that of DMAN at approximately $399.5 \mathrm{eV}$ (II) to a range of from approximately 402 to approximately $403 \mathrm{eV}$ (I). The model used to estimate the energy shifts has already been reported elsewhere. ${ }^{6-8,10,12,14}$ The results for six protonated species of DMAN and the result for nonprotonated DMAN are summarized in Table I.

It can be seen from Table I that the most acidic species experience a slightly higher shift in binding energy. It should also be noted that any change in the acidity of the species being reacted with DMAN seems to influence the complexity of the $\mathrm{H}$ bonded peak structure (i.e., peak I is generally accompanied by a number of additional peaks to create a manifold). This progressive manifold feature appears to relate to the creation of numerous chemistries and structures. The difference between the two peaks that can be deconvoluted from the $\mathrm{N}(1 s)$ peak I manifold may describe the asymmetry of hydrogen bonding formed in proton sponges.

In Fig. 8 the oxygen $1 s$ region for the cryogenically stabilized nonprotonated DMAN shows a single relatively nar- 


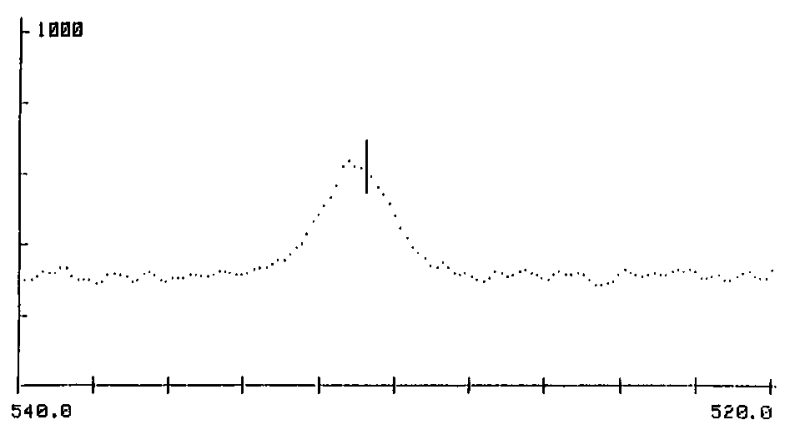

FIG. 8. Oxygen $1 s$ spectra for nonprotonated DMAN.

row peak. Figure 8 is relevant because the absence of a peak at $534 \mathrm{eV}$ (i.e., $\mathrm{OH}$ ) indicates that there was no apparent involvement of water (e.g., ice) with regard to the cryogenically stabilized reference sample. Figure 8 proves that the results have not been affected by water.

Turning now to the condensed phase that formed on the sample probe during the pumping, Fig. 9 is the nitrogen $1 s$ region for the condensed phase material. The condensed phase material was nitrogen rich. The absence of any up-field peaks is evidence of the lack of any hydrogen bonding with the nitrogen. The low nitrogen $1 s$ binding energy for this condensate (compared to the stabilized reference sample) may be due to the operation of the flood gun being more effective with regard to the conformingly deposited condensate.

In addition to the spectral results, several pumping protocols can be gleaned from this study. As noted above, the sample must kept cool enough to prevent it from subliming as the preparation chamber is pumped down. However, the sample probe must also be kept mechanically engaged with the seals to maintain the vacuum and be kept free enough from any condensate in order for the sample to be moved into the analysis chamber when a sufficiently high vacuum is reached. Further, it must be noted that the cold surface of the sample probe can function as a cryotrap, condensing unknown phases that may well begin to out gas as the vacuum level is increased. Perhaps of greatest significance one should note that cryogenic transport may be substituted for

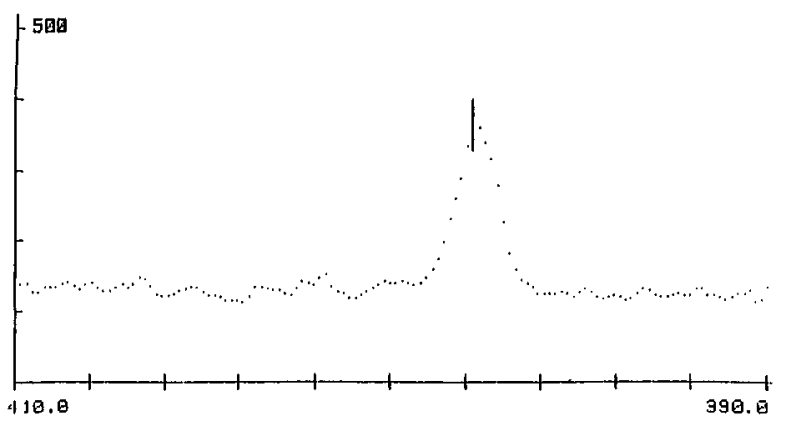

FIG. 9. Nitrogen $1 s$ spectra for condensate phase material. total UHV transport in this case of a vacuum sensitive material without damage of the requisite chemical analysis.

\section{CONCLUSIONS}

DMAN is an excellent model system with which to study consequences of protonation. It is well known that the DMAN molecule sequesters a proton from a countermoiety thus forming a DMANH+ cation with an asymmetric $[\mathrm{N}-\mathrm{H} \ldots \mathrm{N}]+\mathrm{H}$ bonding. If the proton in this bonding were in the center, one would observe just one peak for $\mathrm{N}(1 s)$ electrons. But this is not the case because the proton is closer to one of the nitrogens. This is why one can usually observe a broad complex peak for $\mathrm{N}(1 s)$ electrons, which can be deconvoluted into two peaks for the donor and acceptor nitrogen atoms. As a result the difference in the binding energies for the donor and acceptor can measure the degree of asymmetry of the $[\mathrm{N}-\mathrm{H} \ldots \mathrm{N}]+\mathrm{H}$ bond. The asymmetry of this bond depends on the interactions with the counterion. Because $\mathrm{N}(1 s)$ binding energies of both the donor and acceptor nitrogen atoms are affected, one can compare these values with the binding energies of the unsubstituted DMAN base. Such comparison allows one to estimate the overall effect (the strength) of hydrogen bonding in ionic complexes of proton sponges.

In the case of DMAN, we have shown that the rate of sublimation is temperature sensitive. As a result ESCA spectra have been achieved for DMAN itself, thus permitting an analysis of the total H-bonding effect induced by different acids into this proton sponge. On the basis of these ESCA spectra some new parameters describing the strength and asymmetry of hydrogen bonding can be proposed. Specifically, by comparison of the protonated and nonprotonated forms, one can have a new measure of the strength of hydrogen bonding. Also, the difference between the chemical shifts for $\mathrm{N}$ atoms present in the molecule (which can be obtained when the deconvolution of nitrogen peaks is done) is a good measure of asymmetry of the hydrogen bonding. This makes ESCA spectroscopy useful in studies of weak interactions.

We have shown that the degree of cooling required to stabilize a volatile sample under UHV may be modest and good electron density data from samples that sublime at relatively high pressures (such as those under study here) may be obtained by moderately cooling the sample with inexpensive liquid nitrogen. As a result, the disadvantageous cooling effects on functioning vacuum equipment can be minimized by cooling the sample as little as possible and manipulating any temperature affected parts of the instrument as slowly as possible. This cryogenic stabilization technique may be usefully extended to sample materials that were previously thought to be too difficult to work with, such as phosphorus, or even various phosphoric acids.

In conclusion, it should be noted that the ESCA peak size and shift arguments employed here to define degrees of hydrogen bonding have previously been utilized to describe a variety of additional diamine systems. Interested readers are referred to the appropriate papers. 


\section{ACKNOWLEDGMENTS}

The authors are indebted to Susan J. Kerber, Ph.D., of Material Interface, Sussex, Wisconsin, for her invaluable insights and encouragement.

${ }^{1}$ D. Briggs and M. P. Seah, Practical Surface Analysis, 2nd ed. (Wiley, Chichester, 1990), Vol. 1

${ }^{2}$ T. L. Barr, Modern ESCA (Chemical Rubber, Boca Raton, FL, 1994).

${ }^{3}$ T. L. Barr and S. Seal, J. Vac. Sci. Technol. A 13, 1239 (1995).

${ }^{4}$ G. Barth, R. Linder, and C. Bryson, Surf. Interface Anal. 11, 307 (1988).

${ }^{5}$ S. J. Kerber, J. J. Bruckner, K. Wozniak, S. Seal, S. Hardcastle, and T. L. Barr, J. Vac. Sci. Technol. A 14, 1314 (1996).

${ }^{6}$ K. Wozniak, H. He, J. Klinowski, W. Jones, and T. L. Barr, J. Phys. Chem. 99, 14667 (1995)

${ }^{7}$ K. Wozniak, H. He, J. Klinowski, W. Jones, T. L. Barr, and S. Hardcastle, J. Phys. Chem. 100, 11408 (1996).
${ }^{8}$ K. Wozniak, H. He, J. Klinowski, T. L. Barr, and P. Milart, J. Phys. Chem. 100, 11420 (1996).

${ }^{9}$ J. A. Platts, S. T. Howard, and K. Wozniak, J. Org. Chem. 59, 4647 (1994).

${ }^{10}$ K. Wozniak, J. Mol. Struct. 374, 317 (1996)

${ }^{11}$ K. Wozniak, H. He, J. Klinowski, W. Jones, and E. Grech, J. Phys. Chem. 98, 13755 (1994)

${ }^{12}$ K. Wozniak, H. He, J. Klinowski, and E. Grech, J. Phys. Chem. 99, 1403 (1995).

${ }^{13}$ K. Wozniak, H. He, J. Klinowski, B. Nogaj, D. Lemanski, D. Hibbs, and M. Hursthouse, J. Chem. Soc., Faraday Trans. 91, 3925 (1995).

${ }^{14}$ B. Nogaj, K. Wozniak, D. Lemanski, M. Ostafin, and E. Grech, Solid State Nucl. Magn. Reson. 4, 187 (1995).

${ }^{15}$ K. Wozniak, C. C. Wilson, K. S. Knight, and E. Grech, Acta Crystallogr., Sect. B: Struct. Sci. 52, 691 (1996).

${ }^{16}$ K. Wozniak, T. M. Krygowski, D. Pawlak, W. Kolodziejski, and E. Grech, Phys. Organ. Chem. 10, 814 (1997). 